\title{
A sequence-based hybrid predictor for identifying conformationally ambivalent regions in proteins

\author{
Yu-Cheng Liu ${ }^{\dagger 1}$, Meng-Han Yang ${ }^{\dagger 2}$, Win-Li Lin ${ }^{1}$, Chien-Kang Huang* \\ and Yen-Jen Oyang*2,3,5
}

Addresses: ${ }^{1}$ Institute of Biomedical Engineering, National Taiwan University, Taipei, Taiwan, Republic of China, ${ }^{2}$ Department of Computer Science and Information Engineering, National Taiwan University, Taipei, Taiwan, Republic of China, ${ }^{3}$ Graduate Institutes of Biomedical Electronics and Bioinformatics, National Taiwan University, Taipei, Taiwan, Republic of China, ${ }^{4}$ Department of Engineering Science and Ocean Engineering, National Taiwan University, Taipei 106, Taiwan, Republic of China and ${ }^{5}$ Center for Systems Biology and Bioinformatics, National Taiwan University, Taipei, Taiwan, Republic of China

E-mail: Yu-Cheng Liu - f90548051@ntu.edu.tw; Meng-Han Yang-d95001@csie.ntu.edu.tw; Win-Li Lin - winli@ntu.edu.tw; Chien-Kang Huang* - ckhuang@ntu.edu.tw; Yen-Jen Oyang* - yjoyang@csie.ntu.edu.tw

*Corresponding author †Equal contributors

from Asia Pacific Bioinformatics Network (APBioNet) Eighth International Conference on Bioinformatics (InCoB2009)

Singapore 7-II September 2009

Published: 3 December 2009

BMC Genomics 2009, I0(Suppl 3):S22 doi: 10.1186/1471-2164-10-S3-S22

This article is available from: http://www.biomedcentral.com/I47I-2/64/I0/S3/S22

(c) 2009 Liu et al; licensee BioMed Central Ltd.

This is an open access article distributed under the terms of the Creative Commons Attribution License (http://creativecommons.org/licenses/by/2.0), which permits unrestricted use, distribution, and reproduction in any medium, provided the original work is properly cited.

\begin{abstract}
Background: Proteins are dynamic macromolecules which may undergo conformational transitions upon changes in environment. As it has been observed in laboratories that protein flexibility is correlated to essential biological functions, scientists have been designing various types of predictors for identifying structurally flexible regions in proteins. In this respect, there are two major categories of predictors. One category of predictors attempts to identify conformationally flexible regions through analysis of protein tertiary structures. Another category of predictors works completely based on analysis of the polypeptide sequences. As the availability of protein tertiary structures is generally limited, the design of predictors that work completely based on sequence information is crucial for advances of molecular biology research.

Results: In this article, we propose a novel approach to design a sequence-based predictor for identifying conformationally ambivalent regions in proteins. The novelty in the design stems from incorporating two classifiers based on two distinctive supervised learning algorithms that provide complementary prediction powers. Experimental results show that the overall performance delivered by the hybrid predictor proposed in this article is superior to the performance delivered by the existing predictors. Furthermore, the case study presented in this article demonstrates that the proposed hybrid predictor is capable of providing the biologists with valuable clues about the functional sites in a protein chain. The proposed hybrid predictor provides the users with two optional modes, namely, the high-sensitivity mode and the high-specificity mode. The experimental results with an independent testing data set show that the proposed hybrid predictor is capable of delivering sensitivity of 0.710 and specificity of 0.608 under the high-sensitivity mode, while delivering sensitivity of $0.45 \mathrm{I}$ and specificity of 0.787 under the high-specificity mode.
\end{abstract}


Conclusion: Though experimental results show that the hybrid approach designed to exploit the complementary prediction powers of distinctive supervised learning algorithms works more effectively than conventional approaches, there exists a large room for further improvement with respect to the achieved performance. In this respect, it is of interest to investigate the effects of exploiting additional physiochemical properties that are related to conformational ambivalence. Furthermore, it is of interest to investigate the effects of incorporating lately-developed machine learning approaches, e.g. the random forest design and the multi-stage design. As conformational transition plays a key role in carrying out several essential types of biological functions, the design of more advanced predictors for identifying conformationally ambivalent regions in proteins deserves our continuous attention.

\section{Background}

Proteins are dynamic macromolecules which may undergo conformational transitions upon changes in environment, such as $\mathrm{pH}$, temperature, or upon interactions with other macromolecules [1]. It has been observed in laboratories that conformational transition plays a key role in carrying out several essential types of biological functions, including enzyme catalysis, macromolecule recognition, binding, and signal transduction [2]. For instance, the GTPase HRas protein, whose gene serves as an oncogene of the bladder cancer, shows different conformations in the Switch II region when this protein switches between the RAS-GTP state and the RAS-GDP state [3-6]. Another example is the U1 snRNP A from Homo sapiens. The conformation of one portion of the RNA binding region switches from a helix in the unbound state to a loop in the bound state respectively $[7,8]$. Conformational switches sometimes even cause diseases. For instance, the prion protein ( $\operatorname{PrP})$ causes the mad cow disease when a specific secondary structure element changes from a helix to a $\beta$-sheet [9].

As conformational flexibility is related to protein functions and interactions, scientists have been designing various types of predictors for identifying conformationally flexible regions in proteins [10-12]. In this respect, there are two major categories of predictors. The problem that was firstly addressed by Young et al. [12] concerns identifying polypeptide segments that may fold to form different secondary structure elements in different environments based on sequence analysis. Another major category of the predictors attempts to identify conformationally flexible regions through analysis of protein tertiary structures [2]. As the availability of protein tertiary structures is generally limited, the design of predictors that work completely based on sequence information is crucial for advances of molecular biology research.

In this article, we will propose a novel approach to design a sequence-based predictor for identifying conformationally ambivalent regions in proteins. The novelty in the design stems from incorporating two classifiers based on two distinctive supervised learning algorithms that provide complementary prediction powers. These two machine learning algorithms are the relaxed variable kernel density estimation (RVKDE) algorithm [13,14] and the QUICKRBF algorithm [15] that we have recently proposed. With these two classifiers, the proposed hybrid predictor can operate under either the high-sensitivity mode or the highspecificity mode, depending on the user's application. Experimental results show that the overall performance delivered by the proposed hybrid predictor is superior to the performance delivered by the existing predictors. Furthermore, the case study presented in this article demonstrates that the proposed hybrid predictor is capable of providing the biologists with valuable clues about the functional sites in a protein chain.

\section{Results \\ Overview of the proposed hybrid predictor}

Fig. 1 presents an overview of the structure of the proposed hybrid predictor. The hybrid predictor consists of two classifiers that have been constructed based on two distinctive supervised learning algorithms. As mentioned earlier, the motivation to incorporate two distinctive classifiers was to exploit the complementary prediction powers of alternative supervised learning algorithms. During our study, we observed that for the application addressed in this article the QUICKRBF based classifier consistently delivered sensitivity around 0.7 and specificity around 0.6 regardless of how the usercontrolled parameter was set. Aiming to achieve higher specificity, we therefore investigated the possibility of incorporating two distinctive classifiers with complementary prediction powers. Our study ended up with the design shown in Fig. 1. When the hybrid predictor operates under the high-sensitivity mode, only the QUICKRBF based classifier is enabled. On the other hand, when the hybrid predictor operates under the 


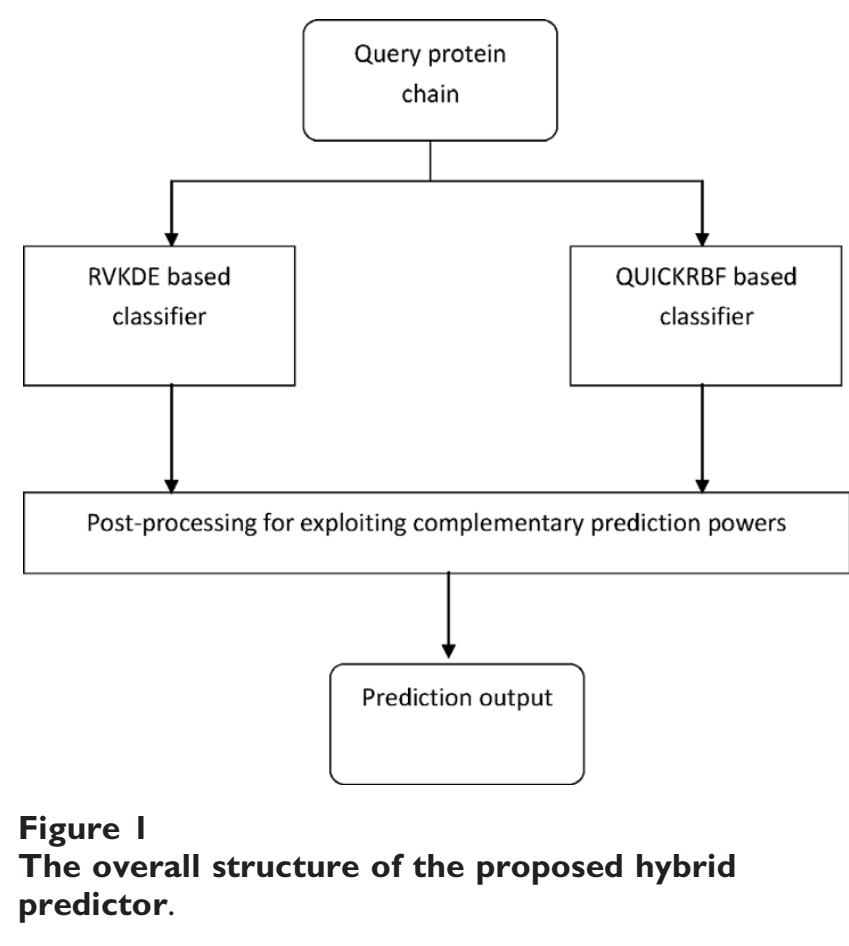

high-specificity mode, both classifiers are enabled and their outputs are merged to achieve higher specificity. With respect to merging the outputs of two classifiers, the following mechanism has been employed. In case the RVKDE based classifier predicts a residue to be conformationally ambivalent but the QUICKRBF based classifier makes an opposite prediction, then the hybrid predictor will check the predictions made by the QUICKRBF based classifier for the four adjacent residues. If three out of the four adjacent residues are marked as conformationally ambivalent, then the concerned residue will be marked as conformationally ambivalent as well. Otherwise, it will be marked as conformationally rigid. Similarly, in case the QUICKRBF based classifier predicts a residue to be conformationally ambivalent but the RVKDE based classifier makes an opposite prediction, then the hybrid predictor will check the predictions made by the RVKDE based classifier for the four adjacent residues. If three out of the four adjacent residues are marked as conformationally ambivalent, then the concerned residue will be marked as conformationally ambivalent as well. Otherwise, it will be marked as conformationally rigid.

The basis of the mechanism described above for merging the outputs of the QUICKRBF based classifier and the RVKDE based classifier is to adopt a more cautious stand in predicting a residue to be conformationally ambivalent. During our study, we tried several alternative mechanisms and decided to employ the one describe above due to its effects observed in the cross validation procedure. The detailed design of QUICKRBF based classifier and the RVKDE based classifier as well as the cross validation procedure employed to set the parameters of the classifiers will be elaborated in the section entitled "Methods".

\section{Generation of the training data set}

Both the learning processes of the RVKDE based classifier and the QUICKRBF based classifier in Fig. 1 have been carried out with the training data set generated by the following procedure.

(1) All the protein chains in the PDB [16] (released on 01-April-2008) that have the same entry name and primary accession number in SwissProt (release 55.1 of 18-March-2008) are grouped. In the end, there are a total of 11084 groups of protein chains.

(2) The BLAST package [17] is invoked to check the redundancy among the groups of protein chains. It is guaranteed that no two protein chains in different groups have a sequence identity higher than $25 \%$. In the end, 3496 groups of protein chains remain.

(3) For each of the 3496 groups of protein chains, the CLUSTALW package [18] is invoked to carry out multiple-sequence alignment on the protein chains in the group and the DSSP package [19] is invoked to label each residue in the protein chains with one of the following 3 types of secondary structure: helix, sheet, and coil. Then, one protein chain is randomly selected from each group as the representative. In this respect, we further checked the sequence identity between the 3496 representatives and the collection of 170 testing protein chains described in the next subsection. We removed 92 representatives due to there existing a homologous testing protein chain with a BLAST-computed sequence identity higher than $20 \%$. Finally, each residue in the remaining 3404 representative protein chains was examined to determine whether the residue and all the residues in other protein chains that are aligned with the residue have been labelled with the same type of secondary structure. A conformationally ambivalent region is defined to be a segment of 3 or more consecutive residues within which each residue and the aligned residues have discrepant types of secondary structures.

(4) The training data set is generated by associating each residue in the 3404 representative protein chains with a feature vector. The feature vectors are derived from the position specific scoring matrices (PSSM) computed by the PSI-BLAST package [20] with window size set to 7. As 
(a)

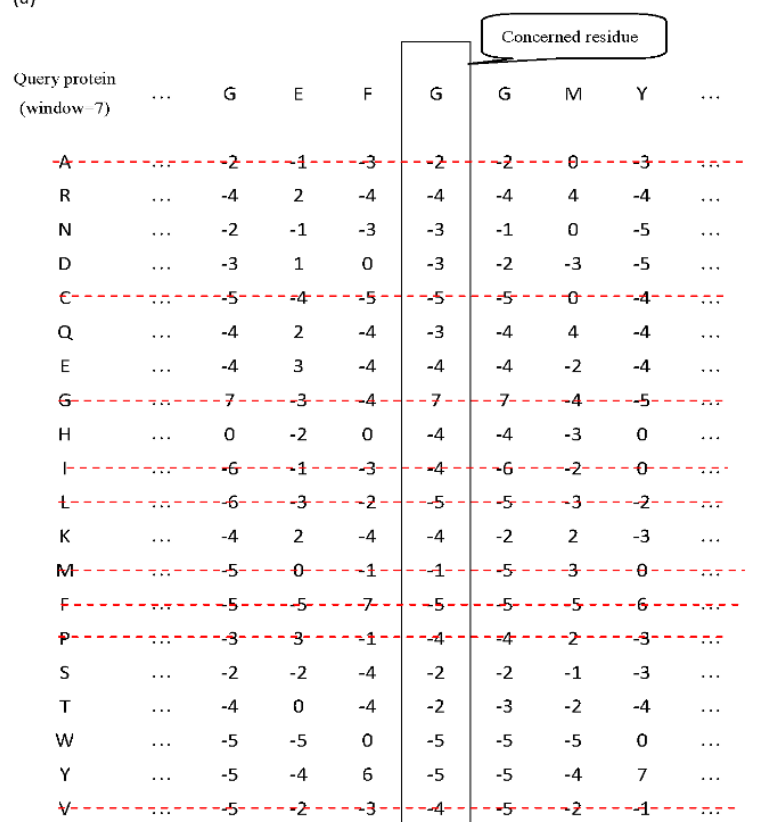

(b)

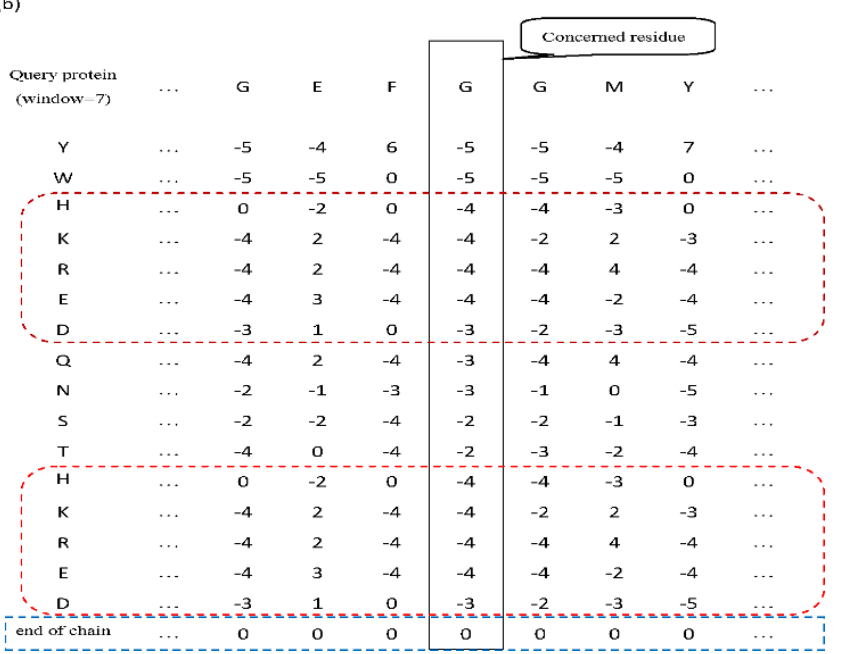

Figure 2

An illustration of the process employed to generate the feature vector of the residue of concern. (a) The feature vectors are derived from PSSM with window size set to 7. Rows corresponding to residue types that are neither charged nor polar are deleted. (b) Rows corresponding to residue types with charge are duplicated and one additional row is included to indicate whether the residue is at one end of the protein chain or not.

illustrated in Fig. 2(a), those rows in the PSSM that correspond to residue types that are neither charged nor polar are deleted. Furthermore, as illustrated in Fig. 2(b), those rows corresponding to residue types with charge are duplicated to increase their influence. Then, a new row is added to record whether the corresponding position is at one end of the protein chain. Finally, the feature vector is generated by concatenating all the rows in the matrix and the values in the feature vectors are scaled to range from 0 to 1 by applying the standard logistic function:

$$
\frac{1}{1+e^{-x}}
$$

One may wonder why we discarded those rows in the PSSM that correspond to residue types that are neither charged nor polar. The reason was that we conducted an analysis on the propensity of residue types in conformationally ambivalent regions and found that the propensity of hydrophobic residues is essentially uniform in conformationally ambivalent regions and in rigid regions. On the other hand, the conformationally ambivalent regions contain significantly higher percentage of charged and/or polar residues than rigid regions. Therefore, we duplicated those rows in the PSSM that correspond to residues with charge.

\section{Generation of the independent testing data set}

The experiments reported in this article have been conducted with an independent testing data set derived from the collection of protein conformational ambivalence regions created by Boden et al. http://pprowler.itee. uq.edu.au/sspred/[10]. According to Boden' s description, this collection of 170 protein chains was extracted from MolMovDB http://www.molmovdb.org/[21,22], which is a database that records the motion of macromolecules, especially proteins, from literatures of PubMed. As mentioned earlier, it was guaranteed that none of these 170 testing protein chains is homologous to the training protein chains described in the previous subsection by having a BLAST-computed sequence identity higher than $20 \%$.

In generating the independent testing data set, we followed the procedure elaborated above for generating the training data set in order to associate each residue in the testing protein chains with a feature vector and labelled each residue as conformationally ambivalent or not based on the annotations in the MolMovDB database. In the end, the testing data set generated contains 5807 positive samples and 54823 negative samples.

\section{Performance metrics}

In this article, the experimental results are reported with the following performance metrics, where TP, TN, FP, and $F N$ represent the numbers of true positive, true negative, false positive, and false negative, respectively. 


$$
\begin{gathered}
\text { accuracy }=\frac{T P+T N}{T P+F P+T N+F N} \\
\text { sensitivity }=\frac{T P}{T P+F N} \\
\text { specificity }=\frac{T N}{T N+F P} \\
\text { precision }=\frac{T P}{T P+F P}
\end{gathered}
$$

Matthews correlation coefficient $(M C C)=\frac{(T P \times T N)-(F P \times F N)}{\sqrt{T P+F N)(T P+F P)(T N+F P)(T N+F N)}}$

$$
\text { F-score }=\left[\frac{1}{2}\left(\frac{1}{\text { sensitivity }}+\frac{1}{\text { precision }}\right)\right]^{-1}
$$

The F-score is the harmonic mean of sensitivity and precision and is a widely used metric in machine learning research for providing a balanced assessment of the performance of a predictor.

\section{Comparison with Boden's predictor of protein conformational ambivalence}

In this section, the performance of the hybrid predictor proposed in this article is compared with that of the predictor proposed by Boden and et al. The approach proposed by Boden is in fact based on a predictor of protein secondary structures [23]. In Boden's approach, if a segment of polypeptide sequence cannot be decisively classified by the predictor of protein secondary structures, then the conformation of this segment is considered to be ambivalent under different environments $[24,25]$. Accordingly, Boden and et al. calculated the entropy associated with each residue in the testing chain based on the probabilities output by the predictor of protein secondary structures. If the entropy of a residue is higher than a cut-off value, then the residue is classified as being in a conformationally ambivalent region. In calculating the entropy of a residue, Boden et al. employed two options of the predictor of protein secondary structures. With the first option, each residue in a protein chain is predicted to belong to one of the following 3 classes of protein secondary structures, alpha-helix, beta-sheet, or coil. On the other hand, with the second option, each residue is predicted to belong to one of the 8 classes of protein secondary structures defined in [19]. Table 1 summarizes the performance delivered by Boden' s 3-class predictor with the independent testing data set. In Table 1, each row corresponds to the performance delivered by the predictor under one specific cut-off value of entropy.

Table 2 shows the performance delivered by the proposed hybrid predictor with the independent testing data set in comparison with Boden' s predictor under the 3 -class mode and the 8-class mode. As mentioned earlier, the hybrid predictor can operate in two modes, namely the high-sensitivity and the high-specifinty mode.

The numbers in Table 2 reveal that when the hybrid predictor and Boden's predictor deliver comparable sensitivity, the hybrid predictor can deliver higher specificity and precision. Furthermore, when the hybrid predictor and Boden's predictor deliver comparable specificity, the hybrid predictor can deliver higher sensitivity and precision.

\section{Comparison with Kuznetsov's predictor of protein conformational ambivalence}

In this section, the performance of the hybrid predictor

\begin{tabular}{|c|c|c|c|c|c|c|c|c|c|c|}
\hline Entropy Cuf-off & TP & $\mathbf{F P}$ & $\mathbf{T N}$ & FN & accuracy & sensitivity & specificity & precision & F-score & MCC \\
\hline 0.05 & 5803 & 54661 & 113 & 0 & 0.098 & 1.000 & 0.002 & 0.096 & 0.175 & 0.014 \\
\hline 0.10 & 5792 & 54364 & 410 & 11 & 0.102 & 0.998 & 0.007 & 0.096 & 0.176 & 0.020 \\
\hline 0.15 & 5703 & 49720 & 5054 & 100 & 0.178 & 0.983 & 0.092 & 0.103 & 0.186 & 0.079 \\
\hline 0.20 & 5572 & 46635 & 8139 & 231 & 0.226 & 0.960 & 0.149 & 0.107 & 0.192 & 0.093 \\
\hline 0.25 & 5328 & 43024 & 11750 & 475 & 0.282 & 0.918 & 0.215 & 0.110 & 0.197 & 0.097 \\
\hline 0.30 & 4903 & 38576 & 16198 & 900 & 0.348 & 0.845 & 0.296 & 0.113 & 0.199 & 0.092 \\
\hline 0.35 & 4468 & 34265 & 20509 & 1335 & 0.412 & 0.770 & 0.374 & 0.115 & 0.201 & 0.088 \\
\hline 0.40 & 4009 & 30101 & 24673 & 1794 & 0.473 & 0.691 & 0.450 & 0.118 & 0.201 & 0.084 \\
\hline 0.45 & 3584 & 26400 & 28374 & 2219 & 0.528 & 0.618 & 0.518 & 0.120 & 0.200 & 0.080 \\
\hline 0.50 & 3142 & 2297I & 31803 & 2661 & 0.577 & $0.54 I$ & $0.58 I$ & 0.120 & 0.197 & 0.073 \\
\hline 0.55 & 2702 & 19722 & 35052 & 3101 & 0.623 & 0.466 & 0.640 & 0.120 & 0.191 & 0.064 \\
\hline 0.60 & 2254 & 16500 & 38274 & 3549 & 0.669 & 0.388 & 0.699 & 0.120 & 0.184 & 0.055 \\
\hline 0.65 & 1730 & 12617 & 42157 & 4073 & 0.724 & 0.298 & 0.770 & 0.121 & 0.172 & 0.047 \\
\hline 0.70 & 1100 & 8011 & 46763 & 4703 & 0.790 & 0.190 & 0.854 & 0.121 & 0.148 & 0.036 \\
\hline 0.75 & 684 & 5183 & 49591 & 5119 & 0.830 & 0.118 & 0.905 & 0.117 & 0.117 & 0.023 \\
\hline 0.80 & 463 & 3443 & 51331 & 5340 & 0.855 & 0.080 & 0.937 & 0.119 & 0.095 & 0.020 \\
\hline 0.85 & 272 & 2238 & 52536 & 5531 & 0.872 & 0.047 & 0.959 & 0.108 & 0.065 & 0.009 \\
\hline 0.90 & 142 & 1339 & 53435 & 5661 & 0.884 & 0.024 & 0.976 & 0.096 & 0.039 & 0.000 \\
\hline 0.95 & 71 & 664 & 54110 & 5732 & 0.894 & 0.012 & 0.988 & 0.097 & 0.022 & 0.000 \\
\hline
\end{tabular}
proposed in this article is compared with that of the sequence-based predictor proposed by Kuznetsov $[11,26]$. It must be noted that Kuznetsov employed a

Table I: Performance of Boden's predictor with different cut-off values of entropy 
Table 2: Performance comparison between the hybrid predictor and Boden's predictor

\begin{tabular}{|c|c|c|c|c|c|c|c|c|c|c|}
\hline Predictor & TP & FP & TN & $\mathbf{F N}$ & accuracy & sensitivity & specificity & precision & F-score & MCC \\
\hline The hybrid predictor (high-sensitivity mode) & 4123 & 21492 & 33331 & 1684 & 0.618 & 0.710 & 0.608 & 0.161 & 0.262 & 0.189 \\
\hline The hybrid predictor (high-specificity mode) & 2617 & 11682 & $43|4|$ & 3190 & 0.755 & 0.451 & 0.787 & 0.183 & 0.260 & 0.165 \\
\hline $\begin{array}{l}\text { Boden's predictor (3-class mode) with entropy } \\
\text { threshold }=0.4\end{array}$ & 4009 & 30101 & 24673 & 1794 & 0.473 & 0.691 & 0.450 & 0.118 & 0.201 & 0.084 \\
\hline $\begin{array}{l}\text { Boden's predictor ( } 3 \text {-class mode) with entropy } \\
\text { threshold }=0.65\end{array}$ & 1730 & 12617 & 42157 & 4073 & 0.724 & 0.298 & 0.770 & 0.121 & 0.172 & 0.047 \\
\hline $\begin{array}{l}\text { Boden's predictor (8-class mode)with entropy } \\
\text { threshold }=0.52\end{array}$ & 2388 & 27895 & 26879 & 976 & 0.503 & 0.710 & 0.491 & 0.079 & 0.142 & 0.094 \\
\hline $\begin{array}{l}\text { Boden's predictor (8-class mode) with } \\
\text { entropy threshold }=0.69\end{array}$ & 1198 & 11563 & 43211 & 2166 & 0.764 & 0.356 & 0.789 & 0.094 & 0.149 & 0.082 \\
\hline
\end{tabular}

different definition of protein conformational ambivalence. By Kuznetsov's definition, a residue in a protein chain is said to be flexible, if its phi degree varies more than 62 or its psi degree varies more than 68 in two different conformations. In order to accommodate Kuznetsov's definition, we labelled the residues in our collection of training protein chains based on Kuznetsov' $s$ definition and then trained our hybrid predictor with this separately-generated training data set.

The testing data set used in this experiment was derived from Boden's collection of testing protein chains. Again, in order to accommodate Kuznetsov's definition of protein conformational ambivalence, we labelled the residues in the testing protein chains based on Kuznetsov's definition. Furthermore, in order to carry out a fair comparison, we removed those testing protein chains in Boden' s collection that are homologous to one or more protein chains in Kuznetsov's training set by having a sequence identify higher than $20 \%$. In the end, 137 out of the 170 testing protein chains in Boden' s collection were used for carrying out the benchmark reported in this section.

Table 3 reports how the hybrid predictor proposed in this article performed in comparison with Kuznetsov's predictor [26]. The numbers in Table 3 reveal that when the parameters of Kuznetsov's predictor and the hybrid predictor are set to deliver comparable levels of specificity, the hybrid predictor can deliver higher sensitivity and precision. It must be noted that in this experiment we carried out an additional run of cross validation to set the parameters of the hybrid predictor differently due to the fact that a different definition of conformational ambivalence is adopted. Furthermore, the results of the proposed hybrid predictor reported in Table 3 include those obtained with only the RVKDE based classifier enabled and those with both classifiers enabled. In this experiment, when both classifiers in the proposed hybrid predictor were enabled, the outputs of these two classifiers were merged based on a slightly different mechanism described in the following. In case the RVKDE based classifier predicts a residue to be conformationally ambivalent but the QUICKRBF based classifier makes an opposite prediction, then the hybrid predictor will check the predictions made by the QUICKRBF based classifier for the four adjacent residues. If any one of the four adjacent residues is marked as conformationally ambivalent, then the concerned residue will be marked as conformationally ambivalent as well. Otherwise, it will be marked as conformationally rigid. Similarly, in the case where the QUICKRBF based classifier predicts a residue to be conformationally ambivalent but the RVKDE based classifier makes an opposite prediction, then the hybrid predictor will check the predictions made by the RVKDE based classifier for the four adjacent residues. If any one of the four adjacent residues is marked as conformationally ambivalent, then the concerned residue will be marked as conformationally ambivalent as well. Otherwise, it will be marked as conformationally rigid.

Table 3: Performance comparison between the hybrid predictor and Kuznetsov' s predictor

\begin{tabular}{|c|c|c|c|c|c|c|c|c|c|c|}
\hline Predictor & TP & $\mathbf{F P}$ & TN & $\mathbf{F N}$ & accuracy & sensitivity & specificity & precision & F-score & MCC \\
\hline $\begin{array}{l}\text { The hybrid predictor with only the RVKDE based } \\
\text { classifier enabled }\end{array}$ & 1236 & 9697 & 34877 & 1033 & 0.771 & 0.545 & 0.782 & 0.113 & 0.187 & 0.166 \\
\hline $\begin{array}{l}\text { The hybrid predictor with both the QUICKRBF } \\
\text { and RVKDE based classifiers enabled }\end{array}$ & 813 & 4792 & 39782 & 1456 & 0.867 & 0.358 & 0.892 & 0.145 & 0.207 & 0.166 \\
\hline $\begin{array}{l}\text { Kuznetsov' s predictor With false positive } \\
\text { rate }=20\end{array}$ & 1020 & 9205 & 35369 & 1249 & 0.777 & 0.450 & 0.793 & 0.100 & 0.163 & 0.126 \\
\hline $\begin{array}{l}\text { Kuznetsov' s predictor With false positive } \\
\text { rate }=10\end{array}$ & 633 & 4676 & 39898 & 1636 & 0.865 & 0.279 & 0.895 & 0.119 & 0.167 & 0.118 \\
\hline
\end{tabular}




\section{Discussion}

Experimental results reported above show that the hybrid approach designed to exploit complementary prediction powers of distinctive supervised learning algorithms works more effectively than the existing predictors. In this section, we will present a real case to demonstrate the effects delivered by the proposed hybrid predictor. Fig. 3(a) depicts two conformations of protein $\mathrm{Ap}_{4} \mathrm{~A}$ hydrolase from Lupinus angustifolius $\mathrm{L}$. One conformation is with ligand $\mathrm{ATP} \cdot \mathrm{MgF}_{\mathrm{x}}$ of which the PDB ID is 1JKN [27], and another conformation is without the ligand, of which the PDB ID is 1F3Y [28]. Fig. 3(b) and 3(c) depict the conformationally ambivalent regions identified in $[27,28]$, and those regions predicted by the proposed hybrid predictor, respectively. It must be noted that in drawing Fig. 3(c) if a gap between two predicted conformationally ambivalent segments contains 4 or fewer residues, then the residues in the gap were also colored as if they were predicted to be in a conformationally ambivalent region. Fig. 3(a), (b), and 3(c) altogether reveal that the conformationally ambivalent regions predicted by the proposed hybrid predictor largely overlap with the structural segments that swing widely in Fig. 3(a). Furthermore, the three predicted conformationally ambivalent regions cover three out of the four conformationally ambivalent regions identified in $[27,28]$. Meanwhile, the only conformationally ambivalent region identified in $[27,28]$ that does not overlap with the predicted conformationally ambivalent regions is the one with the smallest swing, which is colored by blueviolet in Fig. 3(b). As conformational transition plays a key role in carrying out several essential types of biological functions, including enzyme catalysis, macromolecule recognition, binding, and signal transduction, what the case presented in Fig. 3 demonstrates is that the hybrid predictor proposed in this article is capable of providing the biologists with valuable clues about the functional sites in a protein chain.

\section{Conclusion}

In this article, we propose a novel approach to design a sequence-based predictor for identifying conformationally ambivalent regions in proteins. The novelty in the design stems from incorporating two classifiers based on two distinctive supervised learning algorithms that provide complementary predictive powers. Experimental results show that the overall performance delivered by the hybrid predictor proposed in this article is superior to the performance delivered by the existing predictors. Furthermore, the case study presented in this article demonstrates that the hybrid predictor proposed in this article is capable of providing the biologists with valuable clues about the functional sites in a protein chain. (a)

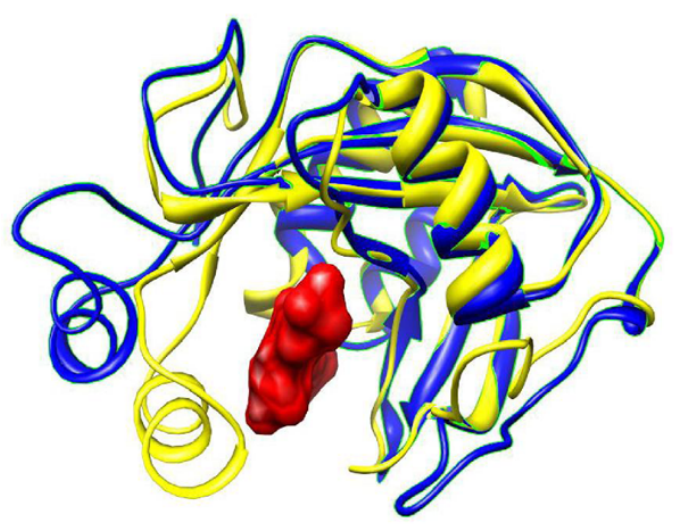

(b)

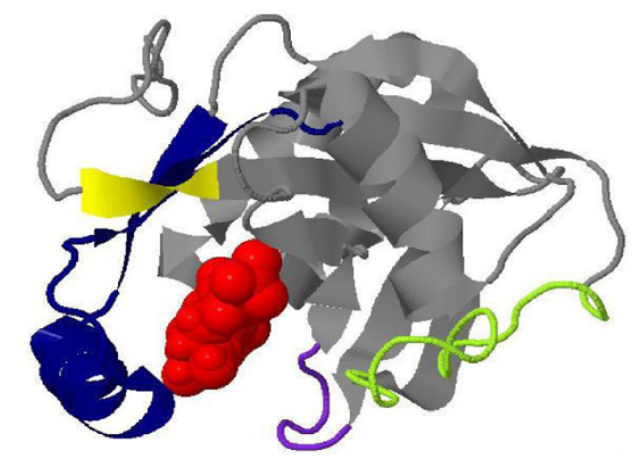

(c)

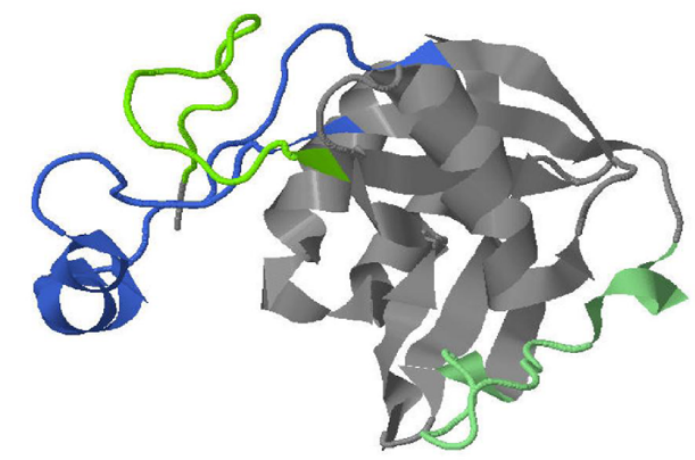

Figure 3

A case study. (a) Two conformations of protein $\mathrm{Ap}_{4} \mathrm{~A}$ hydrolase are plotted by Chimera [36] with different colors. The one colored by yellow is with red-colored ligand ATP. $M g F_{x}$, and the one colored by blue is without the ligand. (b) The conformationally ambivalent regions reported in $[27,28]$ are plotted by Jmol [37] with colors yellow, blueviolet, darkblue, and greenyellow. (c) The conformationally ambivalent regions predicted by the proposed hybrid predictor are plotted by Jmol [37] with colors lawngreen, royalblue, and lightgreen. 
Nevertheless, experimental results also show that there exists a large room for improvement with respect to the performance of the predictor. Therefore, it is of great interest to investigate how to design more advanced predictors. In this respect, it is of interest to investigate how physiochemical properties of polypeptide segments can be more effectively exploited. In this study, we have only exploited the information in the PSSM and a natural extension is to investigate the effects of incorporating the other physiochemical properties of polypeptide segments recently exploited by the related studies [29-31]. Furthermore, it is of interest to investigate the effects of incorporating the lately-developed machine learning approaches, e.g. the random forest design and the multi-stage design [32,33]. As conformational transition plays a key role in carrying out several essential types of biological functions, design of more advanced predictors deserves our continuous attention.

\section{Methods}

\section{Design of the proposed hybrid predictor}

As shown in Fig. 1, the hybrid predictor proposed in this article consists of two classifiers that are constructed with two distinctive supervised learning algorithms. The motivation to incorporate two distinctive classifiers was to exploit the complementary prediction powers of distinctive supervised learning algorithms. Fig. 4 depicts the schematic diagram of a RBF (Radial Basis Function) network for data classification applications. A RBF network consists of three layers, namely the input layer, the hidden layer, and the output layer. The input layer broadcasts the feature vector of the input query sample to each node in the hidden layer. Upon receiving an input vector, each node in the hidden layer then generates an activation based on its associated radial

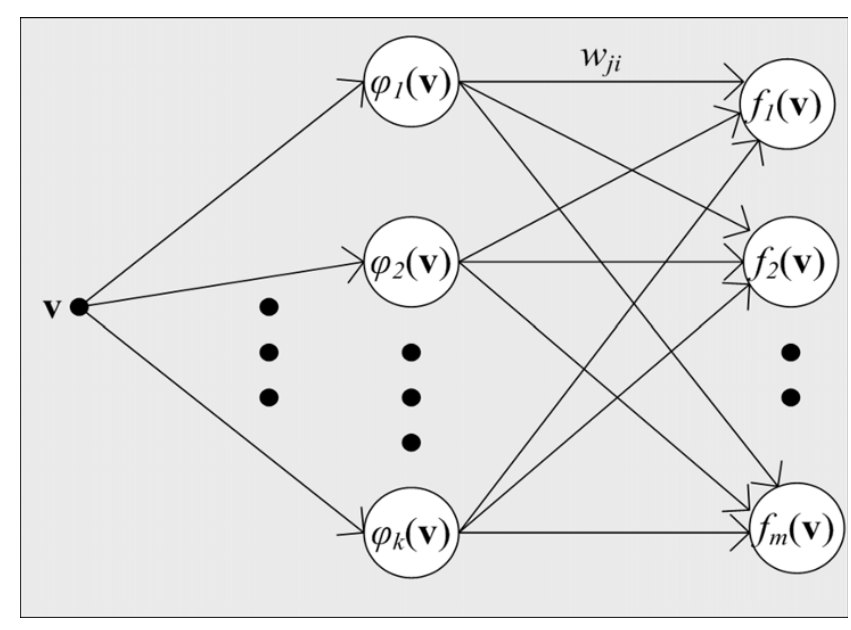

Figure 4

The schematic diagram of a RBF network. basis function $\varphi_{i}(\mathbf{v})$. Finally, each node in the output layer computes a linear combination of the activations generated by the hidden nodes. The general mathematical expression of the output nodes in a RBF network with Gaussian activation functions is as follows:

$$
f_{j}(\mathbf{v})=\sum_{i=1}^{k} w_{j i} \exp \frac{-\left\|\mathbf{v}-\mu_{i}\right\|^{2}}{2 \sigma_{i}^{2}}
$$

where $f_{j}(\mathbf{v})$ is the function corresponding to the $j$-th output node and is a linear combination of $k$ radial basis functions with center $\mu_{i}$ and bandwidth $\sigma_{i} ; w_{j i}$ is the weight associated with the link between the $j$-th output node and the $i$-th hidden node. For data classification applications, the RBF network has one output node corresponding to one class of samples and a query sample is predicted to belong to the class of which the corresponding output node yields the maximum value. The tasks that the learning algorithm of a RBF network carries out include: (1) determining the centers of the activation functions associated with the hidden nodes; (2) setting the parameters associated with the activation functions; (3) optimizing the weights associated with the links between the hidden layer and the output layer.

In our implementation of the QUICKRBF package, the user can specify the number of hidden nodes to be incorporated and then the learning algorithm will place the activation functions at a set of randomly selected training samples. Our experience suggest that how a RBF network performs in terms of classification accuracy is not sensitive to how the bandwidths associated with the activation functions are set, as long as the weights in Equation (1) are optimized. Therefore, the QUICKRBF algorithm simply employs a default value and resorts to the Cholesky decomposition [34] to optimize the weights in Equation (1).

The second classifier in the proposed hybrid predictor is based on the relaxed variable kernel density estimation (RVKDE) algorithm. A kernel density estimator is in fact an approximate probability density function. Let $\left\{\boldsymbol{s}_{1}\right.$, $\left.\boldsymbol{s}_{2}, \ldots, \boldsymbol{s}_{n}\right\}$ be a set of sampling instances randomly and independently taken from the distribution governed by probability density function $f$ in the $d$-dimensional vector space. Then, with the RVKDE algorithm, the value of $f$ at point $\mathbf{v}$ is estimated as follows:

$$
\hat{f}(\mathbf{v})=\frac{1}{|n|} \sum_{s_{i}}\left(\frac{1}{\sqrt{2 \pi} \cdot \sigma_{i}}\right)^{d} \exp \left(-\frac{\left\|\mathbf{v}-s_{i}\right\|^{2}}{2 \sigma_{i}^{2}}\right)
$$

where

1) $\sigma_{i}=\beta \frac{R\left(s_{i}\right) \sqrt{\pi}}{\sqrt[d]{(k+1)} \Gamma\left(\frac{d}{2}+1\right)}$; 
2) $R\left(s_{i}\right)$ is the maximum distance between $s_{i}$ and its $k$ nearest training instances;

3) $\Gamma(\cdot)$ is the Gamma function [35];

4) $\beta$ and $k$ are parameters to be set either through cross validation or by the user.

For data classification applications, one kernel density estimator is created to approximate the distribution of each class of training instances. Then, a query instance located at $\mathbf{v}$ is predicted to belong to the class that gives the maximum value with the likelihood function defined as follows:

$$
L_{j}(\mathbf{v})=\frac{\left|S_{j}\right| \cdot \hat{f}_{j}(\mathbf{v})}{\sum_{q}\left|S_{q}\right| \cdot \hat{f}_{q}(\mathbf{v})},
$$

Where $\left|S_{j}\right|$ is the number of class- $j$ training instances, and $\hat{f}_{j}(\mathrm{v})$ is the kernel density estimator corresponding to class- $j$ training instances. In our current implementation, in order to improve the efficiency of the classifier, we include only a limited number, denoted by $k^{\prime}$, of the nearest class- $j$ training instances of $\mathbf{v}$ while evaluating $\hat{f}_{j}(\mathrm{v})$.

\section{Parameter setting}

As equation (2) exhibits, there are 3 parameters, $\beta, k$, and $k^{\prime}$ associated with a RVKDE based classifier. Supposedly, $d$ should be equal to the dimension of the feature vectors associated with the samples. However, due to the fact that there may exist correlations among features, $d$ is treated as a parameter to be set during the learning process. As a result, to create a RVKDE based classifier, there are a total of 4 parameters to be set. On the other hand, to create a QUICKRBF based classifier, the user only need to determine the number of hidden nodes to be incorporated.

In order to figure out the optimal parameter settings for the proposed hybrid predictor, we have carried out the conventional 5-fold cross validation with the 3404 training protein chains described earlier. The parameters whose values were set with the 5-fold cross validation include the number of hidden nodes in QUICKRBF based classifier, parameters $\beta, k, k^{\prime}$, and $d$ associated with the RVKDE based classifier, as well as the width of the window employed to derive the feature vector of a residue from the PSSM. Table 4 and Table 5 summarize how these parameters have been set in the proposed hybrid predictor. In the experiment conducted to compare the performance of the hybrid predictor and Kuznetsov's predictor, the parameter values shown in
Table 4: Parameter settings of the proposed hybrid predictor for the experiment reported in Table 2

\begin{tabular}{lcccc}
\hline QuickRBF & \multicolumn{4}{c}{ RVKDE } \\
\hline Number of hidden nodes & $d$ & $\beta$ & $k$ & $k^{\prime}$ \\
1400 & $\mathrm{I}$ & 4 & 30 & 200 \\
\hline
\end{tabular}

Table 5: Parameter settings of the proposed hybrid predictor for the experiment reported in Table 3

\begin{tabular}{lcccc}
\hline QuickRBF & \multicolumn{4}{c}{ RVKDE } \\
\hline Number of hidden nodes & $d$ & $\beta$ & $k$ & $k^{\prime}$ \\
1400 & $I$ & 5 & 25 & 190 \\
\hline
\end{tabular}

Table 5 were adopted because a different definition of conformational ambivalence had been employed. In this respect, the parameter values shown in Table 5 were only adopted in this particular experiment and the prediction shown in Fig. 3(c) was obtained with the parameters in the proposed hybrid predictor set in accordance with Table 4.

\section{Competing interests}

The authors declare that they have no competing interests.

\section{Authors' contributions}

MHY and YCL worked together to design the predictor and conducted the experiments. WLL provided many valuable suggestions for this study. $\mathrm{CKH}$ and $\mathrm{YJO}$ proposed and supervised this research. All authors have read and approved the final manuscript.

\section{Note}

Other papers from the meeting have been published as part of BMC Bioinformatics Volume 10 Supplement 15, 2009: Eighth International Conference on Bioinformatics (InCoB2009): Bioinformatics, available online at http://www.biomedcentral.com/1471-2105/10? issue $=\mathrm{S} 15$.

\section{Acknowledgements}

We would like to thank both Dr. Mikael Boden and Dr. Igor B. Kuznetsov for making their benchmark data sets available.

This research has been supported by the National Science Council and National Taiwan University. Funding for open access charge: National Science Council, NSC 97-2627-P-001-002.

This article has been published as part of BMC Genomics Volume 10 Supplement 3, 2009: Eighth International Conference on Bioinformatics (InCoB2009): Computational Biology. The full contents of the supplement are available online at http://www.biomedcentral.com/|47/-2/64/10? issue $=\mathrm{S} 3$. 


\section{References}

I. Creighton TE: Protein folding. Biochem J 1990, 270(I): I-16.

2. Ambroggio $X I$ and Kuhlman B: Design of protein conformational switches. Current opinion in structural biology 2006, I6(4):525-530.

3. Goodsell DS: The molecular perspective: the ras oncogene. The oncologist 1999, 4(3):263-264.

4. Downward J: Targeting RAS signalling pathways in cancer therapy. Nature reviews 2003, 3(I): I I-22.

5. Vetter IR and Wittinghofer A: The guanine nucleotide-binding switch in three dimensions. Science (New York, NY) 200I, 294(5545): I 299-1304.

6. Sprang SR: G proteins, effectors and GAPs: structure and mechanism. Current opinion in structural biology I997, 7(6):849-856.

7. Lutz CS, Cooke C, O'Connor JP, Kobayashi R and Alwine JC: The snRNP-free UIA (SF-A) complex(es): identification of the largest subunit as PSF, the polypyrimidine-tract binding protein-associated splicing factor. RNA (New York, NY) 1998, 4(1 2): | 493-|499.

8. Ellis J and Jones S: Evaluating conformational changes in protein structures binding RNA. Proteins 2008, 70(4): $1518-1526$.

9. Prusiner SB, Scott MR, DeArmond SJ and Cohen FE: Prion protein biology. Cell 1998, 93(3):337-348.

10. Boden $M$ and Bailey TL: Identifying sequence regions undergoing conformational change via predicted continuum secondary structure. Bioinformatics (Oxford, England) 2006, 22(15): 1809-1814.

II. Kuznetsov IB: Ordered conformational change in the protein backbone: prediction of conformationally variable positions from sequence and low-resolution structural data. Proteins 2008, 72(I):74-87.

12. Young M, Kirshenbaum K, Dill KA and Highsmith S: Predicting conformational switches in proteins. Protein Sci 1999, 8(9): I752-|764.

13. Oyang YJ, Hwang SC, Ou YY, Chen CY and Chen ZW: Data classification with radial basis function networks based on a novel kernel density estimation algorithm. leee Transactions on Neural Networks 2005, I 6(I):225-236.

14. Oyang YJ, Ou YY, Hwang SC, Chen CY and Chang DTH: Data classification with a relaxed model of variable kernel density estimation. Proceedings of the International Joint Conference on Neural Networks (IJCNN) 2005, I-5:283 I-2836.

I5. Ou YY, Oyang YJ and Chen CY: $\mathbf{A}$ novel radial basis function network classifier with centers set by hierarchical clustering. Proceedings of the International Joint Conference on Neural Networks (IJCNN), Vols 2005, I-5: I383-1388.

16. Berman HM, Westbrook J, Feng Z, Gilliland G, Bhat TN, Weissig H, Shindyalov IN and Bourne PE: The Protein Data Bank. Nucleic acids research 2000, 28(I):235-242.

17. Altschul SF, Gish W, Miller W, Myers EW and Lipman DJ: Basic local alignment search tool. Journal of molecular biology 1990 , 2I5(3):403-4I0.

18. Thompson JD, Higgins DG and Gibson TJ: CLUSTAL W: improving the sensitivity of progressive multiple sequence alignment through sequence weighting, position-specific gap penalties and weight matrix choice. Nucleic acids research I994, 22(22):4673-4680.

19. Kabsch W and Sander C: Dictionary of protein secondary structure: pattern recognition of hydrogen-bonded and geometrical features. Biopolymers 1983, 22(I 2):2577-2637.

20. Altschul SF, Madden TL, Schaffer AA, Zhang J, Zhang Z, Miller W and Lipman DJ: Gapped BLAST and PSI-BLAST: a new generation of protein database search programs. Nucleic acids research 1997, 25(I 7):3389-3402.

21. Gerstein $M$ and Krebs W: A database of macromolecular motions. Nucleic acids research 1998, 26( I 8):4280-4290.

22. Flores S, Echols N, Milburn D, Hespenheide B, Keating K, Lu J, Wells S, Yu EZ, Thorpe M and Gerstein M: The Database of Macromolecular Motions: new features added at the decade mark. Nucleic acids research 2006, 34 Database: D296-301.

23. Boden M, Yuan Z and Bailey TL: Prediction of protein continuum secondary structure with probabilistic models based on NMR solved structures. BMC bioinformatics 2006, 7:68.

24. Chou PY and Fasman GD: Conformational parameters for amino acids in helical, beta-sheet, and random coil regions calculated from proteins. Biochemistry 1974, I3(2):2 I I-222.

25. Chou PY and Fasman GD: Prediction of protein conformation. Biochemistry 1974, I3(2):222-245.
26. Kuznetsov IB and McDuffie M: FlexPred: a web-server for predicting residue positions involved in conformational switches in proteins. Bioinformation 2008, 3(3): I34-I36.

27. Fletcher JI, Swarbrick JD, Maksel D, Gayler KR and Gooley PR: The structure of $\mathrm{Ap}(4) \mathrm{A}$ hydrolase complexed with ATP-MgF(x) reveals the basis of substrate binding. Structure 2002, I0(2): 205-2I3.

28. Swarbrick JD, Bashtannyk T, Maksel D, Zhang XR, Blackburn GM, Gayler KR and Gooley PR: The three-dimensional structure of the Nudix enzyme diadenosine tetraphosphate hydrolase from Lupinus angustifolius L. Journal of molecular biology 2000 , 302(5): I 165-II77.

29. Tomovic A and Oakeley Ej: Computational structural analysis: multiple proteins bound to DNA. PLOS ONE 2008, 3(9):e3243.

30. Kuznetsov IB and Rackovsky S: On the properties and sequence context of structurally ambivalent fragments in proteins. Protein Sci 2003, I 2(I I):2420-2433.

31. Gunasekaran K and Nussinov R: How different are structurally flexible and rigid binding sites? Sequence and structural features discriminating proteins that do and do not undergo conformational change upon ligand binding. Journal of molecular biology 2007, 365(I):257-273.

32. Hirose $S$, Shimizu K, Kanai S, Kuroda $Y$ and Noguchi T: POODLEL: a two-level SVM prediction system for reliably predicting long disordered regions. Bioinformatics (Oxford, England) 2007, 23( I 6):2046-2053

33. Peng K, Radivojac $P$, Vucetic S, Dunker AK and Obradovic Z Length-dependent prediction of protein intrinsic disorder. BMC bioinformatics 2006, 7:208.

34. Press WH: Numerical Recipes in C.Cambridge: Cambridge University Press; second 1992

35. Artin E: The Gamma Function.New York: Holt, Rinehart and Winston; 1964

36. Pettersen EF, Goddard TD, Huang CC Couch GS, Greenblatt DM, Meng EC and Ferrin TE: UCSF Chimera-a visualization system for exploratory research and analysis. Journal of computational chemistry 2004, 25(13):1605-1612.

37. Jmol: an open-source Java viewer for chemical structures in 3D. http://www.jmol.org.

Publish with BioMed Central and every scientist can read your work free of charge

"BioMed Central will be the most significant development for disseminating the results of biomedical research in our lifetime. "

Sir Paul Nurse, Cancer Research UK

Your research papers will be:

- available free of charge to the entire biomedical community

- peer reviewed and published immediately upon acceptance

- cited in PubMed and archived on PubMed Central

- yours - you keep the copyright
BioMedcentral 